\title{
Massive bleeding despite tourniquet inflation in total knee replacement patient with a femoro-popliteal artery bypass graft
}

\author{
Jung Ho Seok ${ }^{1}$, Yun Hee Lim ${ }^{1,2}$, Byung Hoon Yoo ${ }^{1,2}$, Dong Won Kim², and Tae-in Ham \\ Departments of ${ }^{1}$ Anesthesiology and Pain Medicine, ${ }^{2}$ Thoracic and Cardiovascular Surgery, Sanggye Paik Hospital, \\ ${ }^{3}$ Department of Anesthesiology and Pain Medicne, National Police Hospital, Seoul, Korea
}

A tourniquet is used frequently in orthopedic surgeries, including total knee replacement, to reduce bleeding and enhance the visual field during surgery. Herein, we present a case with massive bleeding that occurred during a total knee replacement that had administered a tourniquet with adequate pressure. A 65-year-old man presented to the orthopaedic clinic for right total knee replacement due to osteoarthritis. The patient had right lower limb artery obstruction and a history of a right femoro-popliteal artery bypass graft 7 years prior to his visit. He was $169 \mathrm{~cm}$ tall weighing $68 \mathrm{~kg}$. The patient had no other specific medical history, and no abnormal finding was covered on the preoperative examination. When the patient arrived at the operating room, his blood pressure was 150/90 $\mathrm{mmHg}$. We provided propofol $120 \mathrm{mg}$ and rocuronium $50 \mathrm{mg}$ followed by induction with sevoflurane and $1: 1$ of oxygen and $\mathrm{N}_{2} \mathrm{O}$. An inflation tourniquet with a pressure of $300 \mathrm{mmHg}$ was applied to the right thigh. While the surgeon was making the skin incision to expose the joint, unpredicted massive bleeding occurred. Just before the tourniquet was applied, the patient's blood pressure was $95 / 65 \mathrm{mmHg}$, and right after the tourniquet was inflated, it was $130 / 80 \mathrm{mmHg}$. Based on the assumption of a tourniquet function error, we applied a new tourniquet with $300 \mathrm{mmHg}$ of pressure on the right thigh. However, the bleeding continued even when we the raised pressure up to $400 \mathrm{mmHg}$. The bleeding decreased considerably when we deflated the cuff. Furthermore, with ultrasonographic guidance, we found that the femoral artery flow was not blocked by the tourniquet; therefore, the surgeon proceeded with the surgery without use of a tourniquet. During the surgery, 1,200 $\mathrm{ml}$ of crystalloid solution, $500 \mathrm{ml}$ of colloid solution and packed RBC 4 units were administered and the estimated blood loss was 2,500 $\mathrm{ml}$. Upon searching for the cause of the unpredicted bleeding, we examined the blood flow of the right femoropopliteal artery bypass graft with ultrasonography 6 months later. It showed that both the femoro-popliteal artery bypass graft with or without $350 \mathrm{mmHg}$ of tourniquet pressure had good blood flow (Fig. 1). A tourniquet is frequently used in total knee joint replacement surgeries to block the vascular flow of the limb during surgery. However, complications such as thigh pain, nerve injury, compartment syndrome, and nonalignment of the patella as well as systemic complications such as deep venous thrombosis, hypertension, and arrhythmia may occur. Therefore, it is important to maintain an adequate pressure and duration while using a tourniquet. There is no absolute limit on the pressure and duration when using a tourniquet, but Flatt [1] reported 2 hours is an adequate time if the patient has no hemodynamic problems. Horlocker et al. [2] reported $300 \mathrm{mmHg}$ for the tourniquet pressure as being adequate for the lower limb. In this case, bleeding continued with an initial tourniquet pressure of $300 \mathrm{mmHg}$ and even continued after we applied $400 \mathrm{mmHg}$ of pressure. The use of the tourniquet instead increased the blood flow in our case. It is believed that the use of the tourniquet was unable to block the graft artery flow but collapsed the venous flow, which resulted in the

Corresponding author: Yun Hee Lim, M.D., Department of Anesthesiology and Pain Medicine, Sanggye Paik Hospital, Sanggye 7-dong, Nowongu, Seoul 139-707, Korea. Tel: 82-2-950-1173, Fax: 82-2-950-1323, E-mail: painfree@paik.ac.kr

(c) This is an open-access article distributed under the terms of the Creative Commons Attribution Non-Commercial License (http:// creativecommons.org/licenses/by-nc/3.0/), which permits unrestricted non-commercial use, distribution, and reproduction in any medium, provided the original work is properly cited. 


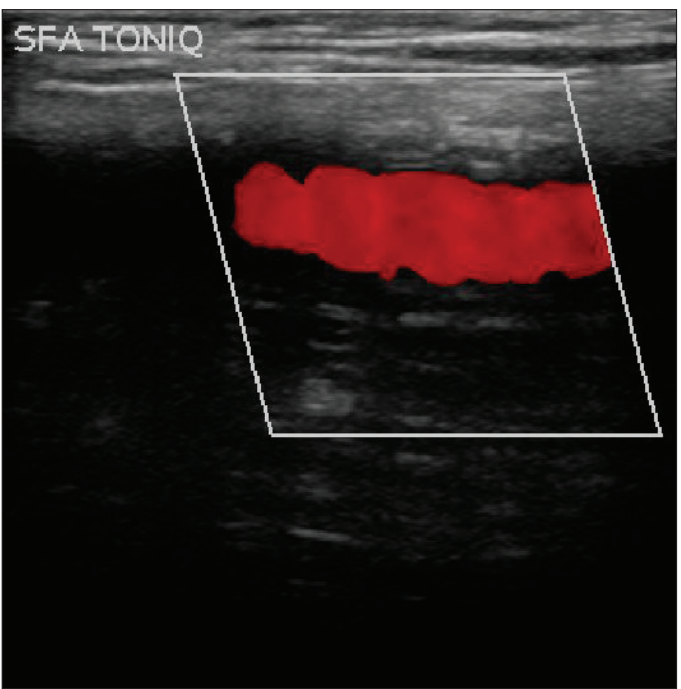

Fig. 1. Doppler sonography of femoro-popliteal bypass graft with tourniquet applying; showing femoro-popliteal bypass graft not collapse.

increased blood loss. In case of artery occlusion above the knee, a polytetrafluroethylene (PTFE) bypass graft is used in elderly when an artery is in poor condition or there is no venous graft. For a PTFE bypass graft, it has good short outcomes compared to the use of a venous graft. For long term outcomes, a PTFE bypass graft shows less patency though. This case also used a PTFE bypass graft and this may have affected the blood flow when compared with a venous graft. However, there is no report on this issue yet. Complications from femoro-popliteal bypass grafts are myocardial ischemia, cerebral infarct, infection on the graft site, peripheral venous thrombosis, and sexual dysfunction by autonomic nerve injury. Infection on the graft site may result in permanent adhesion or fibrosis [3]. Therefore, in our case, it is believed that the patient had an asymptomatic infection, which resulted in adhesion and fibrosis around the artificial vessel graft. The adhesion and fibrosis blocked vessel collapsed even under high pressure causing severe bleeding afterwards. There is controversy about the use of a tourniquet in patients with peripheral artery occlusion. Some say it is safe not to use a tourniquet [4]. Others say there is no difference in postoperative vascular complications by using a tourniquet [5]. Therefore, adequate discussion must be carried out with orthopedists before use of a tourniquet. Patients with a history of a femoro-popliteal bypass graft should be evaluated first with ultrasonography on whether vascular flow can be blocked with a tourniquet. If it is decided that a tourniquet should not be used during surgery, care should be taken for the bleeding during surgery and for postoperative vascular complications.

\section{References}

1. Flatt AE. Tourniquet time in hand surgery. Arch Surg 1972; 104: 190-2.

2. Horlocker TT, Hebl JR, Gali B, Jankowski CJ, Burkle CM, Berry DJ, et al. Anesthetic, patient, and surgical risk factors for neurologic complications after prolonged total tourniquet time during total knee arthroplasty. Anesth Analg 2006; 102: 950-5.

3. Torsello G, Sandmann W. Use of antibiotic-bonded grafts in vascular graft infection. Eur J Vasc Endovasc Surg 1997; 14 Suppl A: 84-7.

4. DeLaurentis DA, Levitsky KA, Booth RE, Rothman RH, Calligaro $\mathrm{KD}$, Raviola CA, et al. Arterial and ischemic aspects of total knee arthroplasty. Am J Surg 1992; 164: 237-40.

5. Smith DE, McGraw RW, Taylor DC, Masri BA. Arterial complications and total knee arthroplasty. J Am Acad Orthop Surg 2001; 9: 253-7. 